\title{
Knockdown of Arabidopsis ROOT UVB SENSITIVE4 Disrupts Anther Dehiscence by Suppressing Secondary Thickening in the Endothecium
}

Running Title: RUS4 is involved in endothecium wall thickening

\author{
Corresponding author: S.-Q. Zhao, Key Laboratory of Chemical Biology and Molecular Engineering \\ of Ministry of Education, Institute of Biotechnology, Shanxi University, Taiyuan, 030006, China. \\ Telephone: +86-351-7011499; Fax: +86-351-7011499; Email: shuqing@,sxu.edu.cn
}

Subject area: growth and development; regulation of gene expression

Number of black and white figures, colour figures, tables and type and number of supplementary material: 9 colour figures, 7 supplementary colour figure and 2 supplementary tables

(C) The Author(s) 2019. Published by Oxford University Press on behalf of Japanese Society of Plant Physiologists. All rights reserved. For permissions, please email: journals.permissions@oup.com 


\section{Knockdown of Arabidopsis ROOT UVB SENSITIVE4 Disrupts Anther Dehiscence by Suppressing Secondary Thickening in the Endothecium}

Running Title: RUS4 is involved in endothecium wall thickening

Shu-Qing Zhao ${ }^{1 *}$, Wen-Chao Li ${ }^{1}$, Yi Zhang ${ }^{1}$, Alison C. Tidy ${ }^{2}$, and Zoe A. Wilson²

${ }^{1}$ Key Laboratory of Chemical Biology and Molecular Engineering of Ministry of Education, Institute of Biotechnology, Shanxi University, Taiyuan, 030006, China

2 School of Biosciences, University of Nottingham, Sutton Bonington Campus, Loughborough, Leicestershire LE12 5RD, United Kingdom

* Corresponding author: E-mail, shuqing@sxu.edu.cn; Fax, +86-351-7011499 


\section{Abstract}

ROOT UV-B SENSITIVE4 (RUS4) encodes a protein with no known function that contains a conserved Domain of Unknown Function 647 (DUF647). The DUF647-containing proteins RUS1 and RUS2 have previously been associated with root UV-B-sensing pathway that plays a major role in Arabidopsis early seedling morphogenesis and development. Here, we show that RUS4 knockdown Arabidopsis plants, referred to as amiR-RUS4, were severely reduced in male fertility with indehiscent anthers.

Light microscopy of anther sections revealed a significantly reduced secondary wall thickening in the endothecium of amiR-RUS4 anthers. We further show that transcript abundance of the $N A C$ domain genes NAC SECONDARY WALL THICKENING PROMOTING FACTOR1 (NST1) and NST2, which have been shown to regulate the secondary cell wall thickenings in the anther endothecium, were dramatically reduced in the amiR-RUS4 floral buds. Expression of the secondary cell wall-associated MYB transcription factor genes MYB103 and MYB85 were also strongly reduced in floral buds of the amiR-RUS4 plants. Overexpression of RUS4 led to increased secondary thickening in the endothecium. However, the rus4-2 mutant exhibited no obvious phenotype. Promoter-GUS analysis revealed that RUS4 promoter was highly active in the anthers, supporting its role in anther development. Taken together, these results suggest that RUS4, probably functions redundantly with other genes, may play an important role in the secondary thickening formation in the anther endothecium by indirectly affecting the expression of secondary cell wall biosynthetic genes.

Key words: anther dehiscence, Arabidopsis thaliana, male sterility, RUS4, secondary wall thickening 


\section{Introduction}

Anther dehiscence and pollen maturation are essential processes occurring late in plant anther development; pollen formation typically involves gene expression from more than half of the genome.

The extent of the genomic involvement in pollen and anther development is illustrated by the high frequency of mutations that result in failure of male fertility, which can be a consequence of impaired pollen development and/or anther dehiscence. Genetic analysis of male sterile mutants in Arabidopsis (Arabidopsis thaliana) and rice (Oryza sativa) has significantly improved the fundamental understanding of pollen and anther development (Wilson and Zhang 2009; Dobritsa et al. 2011, Cui et al. 2012, Quilichini et al. 2015).

Pollen development occurs within the anther, which contains four cell layers, the epidermis, endothecium, middle layer and tapetum (Scott et al. 2004, Zhang and Yang 2014). Endothecium development is coordinated with pollen maturation and the degeneration of the anther tapetum and middle cell layer. The anther endothecium and the localized secondary thickening within this cell layer play a critical role in anther dehiscence and pollen release, as it is responsible for providing the mechanical force for anther opening (Keijzer 1987, Bonner 1989, Wilson et al. 2011, Nelson et al., 2012). The basic process of anther dehiscence appears highly conserved among different plant species, with the dicot model plant Arabidopsis thaliana (Sanders et al. 1999) showing a similar dehiscence process to the Solanaceae (Bonner and Dickinson 1989, Sanders et al. 2005) and to monocots rice and maize (Matsui et al., 1999, Keijzer et al., 1996). The main events of the dehiscence program include: the formation of fibrous bands on the endothecial cell walls, degeneration of the septum cells leading to a bilocular anther, and finally breakage of the stomium and release of pollen grains (Goldberg et al. 1993). 
Multiple studies on NAC and MYB transcription factors have identified a transcriptional network regulating secondary wall biosynthesis (Mitsuda et al. 2005, Mitsuda et al. 2007, Zhong et al. 2008). Two NAC transcription factors, NAC SECONDARY WALL THICKENING PROMOTING FACTOR1 (NST1) and NST2, have been shown to function redundantly in regulating secondary wall thickening in anther endothecium (Mitsuda et al. 2005). Transgenic plants expressing the chimeric NST1 and NST2 repressors have defects in anther dehiscence due to a loss of secondary wall thickening in the anther endothecium (Mitsuda et al. 2005). In addition to NACs, several MYB transcription factors have also been shown to be important regulators of secondary wall biosynthesis in Arabidopsis. MYB26 has been suggested to function as an initial switch regulating secondary wall formation in the anther endothecium, acting by directly regulating NST1 and NST2 (Yang et al. 2007, Yang et al. 2017). However, a recent study has shown that the downstream NST1 and NST2 factors are insufficient for secondary thickening formation in the anther and that expression of MYB26, and presumably the equivalent regulator in the vegetative tissues, is essential for correctly localized secondary thickening formation (Yang et al. 2017). MYB103 was initially identified as a member of the transcriptional network regulating secondary wall biosynthesis in fiber cells of Arabidopsis (Zhong et al. 2008). It was shown to be a direct transcriptional target of NST1 and NST2 (Zhong et al. 2008), and is required for biosynthesis of syringyl lignin in the Arabidopsis inflorescence stem (Öhman et al. 2013). Another transcription factor MYB85 has also been shown to be involved in regulating secondary cell wall biosynthesis in Arabidopsis (Zhong et al. 2008).

The deposition of lignified secondary thickening usually occurs when cell growth has stopped. Secondary cell walls are composed predominantly of cellulose, lignin, and xylan, and the biosynthesis and deposition of wall material involves the coordinated regulation of a number of complex metabolic 
pathways (Zhong and Ye 2015, Zhong et al. 2018). The cellulose synthase (CESA) enzymes, also called IRREGULAR XYLEM (IRX), are essential components of the cellulose synthesizing complex required for secondary cell wall synthesis. Mutation of any of the three Arabidopsis secondary wall CESA genes, CESA4/IRX5, CESA7/IRX3 and CESA8/IRX1, results in a severe reduction in secondary cell wall cellulose, leading to weakened stems, with reduced mechanical strength and an irregular xylem phenotype (Turner and Somerville 1997, Taylor et al. 1999, Taylor et al. 2000, Taylor et al. 2003, Brown et al. 2005). The GAUT1-related gene family member GAUT12/IRX8 is a putative galacturonosyltransferase involved in Arabidopsis secondary cell wall biosynthesis, integrity and anther dehiscence (Persson et al. 2007, Hao et al. 2014). Multiple studies have shown that mutants defective in endothecium secondary thickening exhibit altered expression of these CESA/IRX genes (Mitsuda et al. 2005, Yang et al. 2007, Jung et al. 2013, Kim et al. 2012).

The Arabidopsis genome encodes six Domain of Unknown Function 647 (DUF647) family members, namely ROOT ULTRAVIOLET B-SENSITIVE1 (RUS1, At3g45890), RUS2 (At2g31190), RUS3 (At1g13770), RUS4 (At2g23470), RUS5 (At5g01510) and RUS6/EMB1879 (At5g49820) (Tong et al. 2008, Leasure et al. 2009). Two of these, RUS1/WXR3 and RUS2/WXR1, have been shown to play vital roles in Arabidopsis early seedling morphogenesis and development; they work together in Arabidopsis root UV-B-sensing pathways and polar auxin transport (Tong et al. 2008, Leasure et al. 2009, Ge et al. 2010, Yu et al. 2013). However, the biological functions of the remaining four members of the DUF647 family have not yet been characterized.

In this study, we performed a functional characterization of the DUF647 family gene RUS4 in Arabidopsis using reverse genetics. Artificial microRNA (amiRNA) knockdown RUS4 plants, referred to as amiR-RUS4, exhibited severely reduced male fertility with defects in anther dehiscence. 
Microscopic analysis revealed that anthers from the amiR-RUS4 plants had defective endothecium secondary thickening. Several genes associated with secondary cell wall thickening showed decreased expression in amiR-RUS4 flower buds. Overexpression of RUS4 led to increased secondary wall thickening in the endothecium; however, the knockout mutant of RUS4 exhibited no obvious phenotype. We further showed that the RUS4 promoter is highly active in anthers, supporting its role in anther development. Taken together, our results suggest that RUS4 may play an important role in anther development in Arabidopsis, through its impact on the process of secondary wall formation in the endothecium. It is likely that RUS4 functions redundantly with other genes, possibly including the DUF647 family member RUS6. These findings suggest much wider roles for the DUF647 domain family members in plant growth and development, beyond root UV-B sensing.

\section{Results}

Knockdown of RUS4 leads to a severe reduction in male fertility

For functional analysis of the previously uncharacterised DUF647 family genes, we designed an artificial microRNA (amiRNA) targeting the Arabidopsis DUF647-containing gene RUS4 (Fig. 1A). Upon constitutive expression of the RUS4-amiRNA construct in Arabidopsis, RUS4 expression was down-regulated in the transgenic lines. Three independent homozygous RUS4 knockdown lines were identified (Supplementary Fig. S1A). These lines exhibited normal vegetative growth, but showed severely reduced fertility (Fig. 1C; Supplementary Fig. S1B). We did not observe particular fertility differences among these lines, which is consistent with the similar degree of RUS4 mRNA reduction (Supplementary Fig. S1A). The RUS4-amiRNA transgenes were stably inherited, and they remained effective in the progeny. One representative line (line 1), which was stable for more than five 
generations, subsequently referred to as amiR-RUS4, was characterized in detail.

At six weeks old, wild-type plants exhibited many fully elongated siliques filled with developing seeds, whereas the equivalent amiR-RUS4 plants produced short siliques without seeds (Fig. 1C, arrow), although occasional siliques with developed seeds were observed as the plants aged.

(Supplementary Fig. S1B, arrows). These seeds were not a result of cross pollination, as the sterility phenotype was maintained in the progeny from these seeds and was identical with that of the homozygous paternal amiR-RUS4 plants. The average seed yield of the amiR-RUS4 plants was approximately $7.3 \%$ of that of wild-type (Fig. 1F).

To determine the nature of the observed sterility, we performed reciprocal crosses between wild-type and amiR-RUS4 plants. It was possible to fertilize the amiR-RUS4 flowers using wild-type anthers, whereas pollination of wild-type flowers with amiR-RUS4 anthers did not result in seed production (Fig. 1D). These results demonstrate that male fertility is impaired; whilst no detectable effect was seen in the reciprocal crosses when using wild-type plants as a pollen donor, indicating that female fertility is unaffected in the amiR-RUS4 plants.

To confirm that the reduced fertility was caused by the reduction of RUS4 expression, we cloned RUS4 cDNA from developing flower buds in order to complement the amiR-RUS4 plants. The construct containing RUS4 cDNA driven by the CaMV $35 \mathrm{~S}$ promoter (35S:RUS4) was generated and transformed into the homozygous amiR-RUS4 plants. The homozygous T2 transgenic amiR-RUS4 line carrying the wild-type RUS4 cDNA transgene showed increased RUS4 expression (Fig. 1B) and restored the fertility to wild-type levels, with numerous fully developed siliques (Fig. 1E, arrow). We therefore concluded that knockdown of RUS4 gene in amiR-RUS4 plants was responsible for the reduced fertility observed. 
To identify Arabidopsis T-DNA insertion mutants at the RUS4 locus, we screened the ABRC

collections and carried out phenotypic analysis of the identified insertion mutants. Two independent T-

DNA lines, GK-345D06 and GK-447F02, designated rus4-1 and rus4-2, carrying a T-DNA insertion

either at the promoter or in the second exon, respectively (Supplementary Fig. S2A), were genotyped

using PCR-based analysis of genomic DNA. Expression analysis of the homozygous rus4-1 mutant

exhibited an increase of RUS4 transcript; RUS4 transcript could not be detected in the homozygous

rus4-2 mutant (Supplementary Fig. S2B), indicating that it contains a null mutation in the RUS4 gene.

Nevertheless, both rus4-1 and rus4-2 mutants were fertile and indistinguishable from the wild type

under our growth conditions (Supplementary Fig. S2C). This discrepancy of fertility phenotype

between rus 4-2 mutant and amiR-RUS4 knockdown line could be explained by several reasons,

including amiRNA off-target effects or genetic compensation induced by RUS4 knockout mutation.

To test whether the expression of the other genes of DUF647 family were altered in the amiR-RUS4 line and the null mutant rus4-2, we performed a comparative qRT-PCR analysis of the expression of all six RUS genes in flower buds of wild-type, amiR-RUS4 and rus4-2 plants. Our results showed that the other $R U S$ genes were not significantly changed in the amiR-RUS4 line, except for the down-regulation of RUS4 (Supplementary Fig. S2D), indicating that the RUS4-amiRNA construct is acting specifically on RUS4. However, the rus4-2 mutant exhibited a slight upregulation of RUS6 (Supplementary Fig. S2D), suggesting its possible role in compensation for the loss of RUS4 function.

\section{The RUS4 gene expression pattern}

To study the RUS4 gene expression pattern, we first measured the relative levels of RUS4 transcripts in various Arabidopsis tissues using quantitative RT-PCR. Although RUS4 transcript could be detected in various tissues, the expression was higher in the closed flower buds, open flowers and vegetative rosette 
leaves (Fig. 2A). Next, we generated a RUS4pro:GUS construct and introduced it into wild-type Col-0 Arabidopsis. Out of four homozygous transgenic lines, which showed similar GUS staining patterns, we chose one representative line for detailed study. When the inflorescences of RUS4pro:GUS plants were stained for GUS activity, GUS signal was not detected in buds younger than stage 9 (Fig. 2B). GUS staining was first visualized in the anthers at flower stage 9 (Fig. 2C, arrow), and became strong in anthers at stage 10 (Fig. 2C, arrowhead). GUS activity reached the highest level in anthers at stages 11 and 12 (Fig. 2D, E), and remained relatively high in stage 13 anthers (Fig. 2F). The mature, released pollen also showed GUS staining (Fig. 2F, arrow; Fig. 2G). A closer examination of anthers from stage 10 buds revealed that GUS activity was extremely high within the tapetum, and was also visible in the endothecium and developing pollen grains (Fig. 2H). Examination of the GUS stained anthers at stage 11 revealed that the strong GUS signal was due to substantial RUS4 expression in pollen grains located inside the anther, and also to expression in the endothecium, and GUS staining was visible in the vascular bundle of anther filament (Fig. 2I). In addition, weak GUS staining was observed in vascular tissues of stems, hypocotyls and roots (Supplementary Fig. S3A-C). In the stem sections, GUS activity was detected in the epidermis, xylem elements and interfascicular fibers (Supplementary Fig. S3D). However, GUS staining was not detected in siliques containing developing seeds, petals (Supplementary Fig. S3E), and seedlings of RUS4pro:GUS lines (Supplementary Fig. S3F). The RUS4 gene was not ubiquitously expressed; instead, its expression is temporally and spatially restricted in the anthers of the later stages of pollen formation, and preferentially localized in organs with relatively large proportions of cells with secondary walls, suggesting that RUS4 may function primarily in the late stages of anther development, and may be associated with secondary wall formation. 
Observation of the amiR-RUS4 flowers at stage 13 showed that the flowers were of normal size and shape, the papillae of the stigmas developed fully, and the filaments of the stamens elongated as in the wild-type flowers (Fig. 3A, B). However, anthers from amiR-RUS4 open flowers failed to dehisce and release pollen grains (Fig. 3B), whereas equivalent stages of wild-type anthers were fully dehisced with an abundance of released pollen (Fig. 3A). We further examined the release of pollen by placing whole mature anthers from wild-type and amiR-RUS4 flowers with the open anther locules facing down on top of a water droplet as described previously by Jakobsen et al. (2005). In the amiR-RUS4 anthers, no pollen release was observed from 32 anthers analyzed, whereas pollen was released from all of the 35 wild-type anthers tested (Fig. 3C, D).

Observation of whole-mount cleared mature stigmas by differential interference contrast (DIC) microscopy showed that when receptive, the stigmatic papillae of wild-type were covered with pollen grains (Fig. 3E, arrow), whereas no pollen grains were observed on the stigmatic papillae of the amiRRUS4 plants (Fig. 3F).

Scanning electron microscopy (SEM) analysis of anther morphology confirmed the defect in anther dehiscence in the amiR-RUS4 plants; at stage 13, wild-type anthers split open along the stomium (Fig. 3G), whereas amiR-RUS4 anthers remained intact (Fig. 3H). Although occasional cleavage at the stomium could be observed in the amiR-RUS4 anthers, the pollen grains generally remained encased within the locules (Fig. 3I). As a result, stigmas with normally expanded papillae did not receive pollen grains. These observations indicate that anther dehiscence is impaired in amiR-RUS4 plants, resulting in defective pollination, and consequently, male sterility.

The amiR-RUS4 anthers are defective in endothecium secondary thickening 
To investigate the dehiscence defects in more detail, we examined transverse sections of amiR-RUS4 anthers at different developmental stages. The anther development appeared to follow the same pattern in amiR-RUS4 and wild-type anthers up to stage 10, with the anthers developing the correct number of cell layers, forming the uninucleate microspores, and degenerating tapetum as in the wild-type (Fig. 4A, B). However, abnormalities in amiR-RUS4 anthers were apparent in comparison with wild-type starting from anther stage 11. For the wild-type at anther late stage 11, the endothecial cell layer appeared fully expanded, which was evident as a radial rectangle cells layer, and fibrous bands of secondary thickening had appeared in the endothecial cells; these processes occurred concurrently with the initial steps of septum degeneration and stomium differentiation (Fig. 4C). The cell differentiation events of the amiRRUS4 plants at anther stage 11, including septum cell degeneration and stomium cell differentiation, were similar to those seen in wild-type (Fig. 4D). However, there was a significant reduction of secondary thickening observed in the amiR-RUS4 endothecium, with only occasional endothecial cells showing fibrous bands (Fig. 4D, labelled Fb). From stage 12 to 13, the fibrous bands in wild-type endothecium became progressively more pronounced (Fig. 4E, G). At stage 12, the pollen grains were still contained within the anther locule, and the anther became bilocular after degeneration and breakage of septum below the stomium in the wild-type anthers (Fig. 4E). In contrast, only a few fibrous bands were visible in amiR-RUS4 endothecial cells. However, the degeneration and breakage of the septum occurred normally as in the wild-type, resulting in a bilocular anther (Fig. 4F). During stage 13-14, pollen grains were released from the locules of wild-type anthers as a consequence of both stomium degeneration and the forces generated by anther dehydration and the secondary wall thickening in the endothecium (Fig. $4 \mathrm{G}$ ); whereas degeneration of the stomium and release of pollen did not occur in the amiR-RUS4 anthers (Fig. 4H). During stage 14, shrinkage of the anther cell layers occurred in a similar manner in amiR- 
RUS4 and wild-type anthers, but the fibrous bands in the endothecium were still only evident in wildtype anthers (Fig. 4I). Breakage of the amiR-RUS4 stomium region within the epidermal layer did not occur and pollen was not released (Fig. 4J).

To further determine the nature of the defects in the endothecium secondary wall thickening in the amiR-RUS4 anthers, we investigated the lignified materials that are major components of secondary cell walls. In the wild-type, staining with ethidium bromide/acridine orange confocal microscopy indicated secondary thickening in the endothecium as bands of striated spring-like thickening (Fig. 5A, D). In contrast, only few isolated lignified cells (Fig. 5B, E) were observed in the endothecium of the amiRRUS4 anthers. These observations indicate that anther indehiscence of the amiR-RUS4 plants was due to defective secondary wall thickening in the endothecial cells.

Knockdown of RUS4 alters the expression of several genes associated with secondary wall thickening It has been previously shown that two NAC domain genes, NST1 and NST2, regulate secondary thickening in anther endothecium (Mitsuda et al. 2005). We therefore examined the transcript abundance of NST1 and NST2 in flower buds of wild-type and the amiR-RUS4 plants at various developmental stages by qRT-PCR. The expression of NST1 and NST2 was negligible in wild-type flower buds at stage 9 and younger, high levels of expression were detected at stage 10, with maximum expression observed at stage 11 when secondary thickening is deposited, expression then decreased dramatically at stage 12 (Fig. 6A, B). In contrast, the transcript abundance of $N S T 1$ and NST2 was dramatically reduced in the amiR-RUS4 flower buds, with only very low levels of expression seen from stages younger than 9 to 12 , and no peak of expression at stages 10 and 11, unlike that seen in the wild-type (Fig. 6A, B).

We also analyzed the expression of secondary cell wall-associated MYB transcription factor genes MYB103 and MYB85 in flower buds of wild-type and the amiR-RUS4 plants at various developmental 
stages by qRT-PCR. We found that the transcript abundance of both genes was strongly decreased in floral buds of the amiR-RUS4 plants (Fig. 6C, D). We next examined the expression of the secondary cell wall-specific cellulose synthase genes CESA4/IRX5, CESA7/IRX3 and CESA8/IRX1, and the galacturonosyltransferase gene GAUT12/IRX8 using qRT-PCR. Our results showed that the amiRRUS4 plants displayed a similar reduction in expression of the CESA4, CESA7, CESA8 and GAUT12 as compared with the wild-type. The wild-type peak of CESA genes and GAUT12 expression at stage 11 did not occur in the amiR-RUS4 plants, with the expression levels reduced by over $85 \%$ in amiR-RUS4 plants as compared with the wild-type (Fig. 6E-H), suggesting that cellulose and xylan biosynthesis were inhibited in amiR-RUS4 flower buds.

RUS4 affects endothecium secondary thickening, but appears not to be directly associated with the regulation of secondary thickening by MYB26

The transcription factor MYB26 has been shown to regulate the formation of secondary thickening in the endothecium (Yang et al., 2007; Yang et al., 2017), we therefore examined the transcript abundance of MYB26 in flower buds of the amiR-RUS4 plants at various developmental stages by qPCR. We found that MYB26 was expressed at a low level in wild-type flower buds until stage 10 when it peaked before tapetum degeneration, and then decreased dramatically at stage 11 when endothecium lignification occurs, and was negligible at stage 12 (Supplementary Fig. S4A). Unexpectedly, MYB26 showed an increased and prolonged expression in amiR-RUS4 floral buds as compared to the wild-type; even at stage 11, while MYB26 was expressed at a very low level in wildtype flower buds, MYB26 expression remained relatively high in amiR-RUS4 flower buds (Supplementary Fig. S4A). This result suggests that RUS4 may affect endothecium secondary thickening via an alternative, potentially novel, pathway independent of MYB26. To test this 
possibility, we examined the effect of MYB26 mutation on RUS4 expression by quantitative RT-PCR.

The result indicates that RUS4 transcript levels are similar in wild-type and myb26 mutant flower buds (Supplementary Fig. S4B).

To further investigated the genetic relationship between RUS4 and MYB26. The 35S:RUS4 construct described previously was introduced into the $m y b 26$ background to determine whether overexpression of RUS4 would suppress the myb26 mutant phenotype. RT-PCR showed that RUS4 expression was increased in homozygous 35S:RUS4/myb26 transgenic plants (Supplementary Fig. S4C). However, there was no rescue of the myb26 sterility phenotype (Supplementary Fig. S4D), suggesting that while RUS4 affects endothecium secondary thickening appears not to be directly associated with the regulation by MYB26, the presence of MYB26 is still required for normal fertility.

Overexpression of RUS4 leads to enhanced secondary thickening in the endothecium

To further test the effect of RUS4 on endothecium secondary thickening, we used a gain-of-function approach. The previously described construct containing RUS4 cDNA driven by the CaMV $35 \mathrm{~S}$ promoter was also transformed into the wild-type Arabidopsis Col-0 plants. Transgenic lines were tested by qRT-PCR to identify those showing overexpression of RUS4 (Fig. 7A). We found that the RUS4-overexpression line (RUS4-OE) showed significantly increased expression of NST1 in floral buds at stages 10 and 11 (Fig. 7B), when NST1 are most highly expressed in wild-type flower buds according to above analysis (Fig. 6A). Although NST2 expression was not changed at stage 10, it increased significantly at stage 11 (Fig. 7C). It seems that NST1 expression is first activated, and then causes up-regulation of NST2. We also analyzed the transcript levels of MYB103 and MYB85, which have been shown to be the downstream targets of NST1 and NST2 (Zhong et al. 2008). Our results showed that MYB103 and MYB85 transcript levels were significantly increased in flower buds of the 
RUS4-overexpression line (Fig. 7D, E). As expected, the RUS4-OE lines exhibited enhanced secondary thickening in the endothecium (Fig. 5C, F; Supplementary Fig. S5B-D, F-H), and also showed increased thickening in the filaments (Fig. 5I; Supplementary Fig. S5J-L); however, the levels of secondary thickening in the leaves and stems of the RUS4-OE lines appeared not significantly different from that of wild-type (Supplementary Fig. S6B-D; F-H), possibly due to the relative low level of induction of NST1/2 expression.

Overexpression of NST1 and NST2 cannot rescue the anther indehiscence and sterility phenotype of amiR-RUS4 plants

To investigate whether overexpression of NST1 and NST2 could suppress the loss-of-function phenotype of amiR-RUS4 plants, we expressed NST1 and NST2 in amiR-RUS4 plants under control of the strong CaMV 35S promoter (35S:NST1 and 35S:NST2). First, we tested whether the overexpression constructs could complement the amiR-RUS4 male sterile phenotype. The homozygous transgenic lines showed a significant increase in NST1 and NST2 transcript levels, respectively (Supplementary Fig. S7A, B). We found that the 35S:NST1 and 35S:NST2 transgenic lines in the amiR-RUS4 background had stunted growth. The retardation of plant growth and development was more severe in the 35S:NST1 transgenic plants than in 35S:NST2 transgenic plants (Supplementary Fig. S7C, D). Furthermore, the fertility of both 35S:NST1 and 35S:NST2 transgene plants in the amiR-RUS4 background was not rescued (Supplementary Fig. S5E, F). Next, we dissected stage-13 flowers and observed the anther dehiscence phenotype of the transgenic lines. Again, there was no difference in anther dehiscence between 35S:NST1/amiR-RUS4, 35S:NST2/amiR-RUS4 and the amiR-RUS4 plants (Supplementary Fig. S7H-J). The lack of rescue of amiR-RUS4 phenotype by overexpression of NSTI and NST2 indicates that RUS4 is required for anther dehiscence, and hence normal fertility, through 
indirect effects on NST1 and NST2 action, in some way other than by epistatic interactions.

\section{RUS4 is localized to the chloroplast}

To characterize the subcellular localization of RUS4, we introduced the translational fusion 35Spro:RUS4-GFP into Arabidopsis amiR-RUS4 as well as into wild-type Col-0 plants. Chloroplast localization of RUS4 has previously been predicted (Kaundal et al., 2010), and consistent with this, stable expression of 35Spro:RUS4-GFP in amiR-RUS4 as well as wild-type Col-0 plants resulted in a signal highly localized to the chloroplasts in the young rosette leaves (Fig. 8A-C), and hypocotyls (Fig. 8D-E), confirming that the protein is chloroplast localized. The chloroplast-localization of RUS4 implicates that its effect on secondary cell wall thickening in the endothecium might be mediated by plant hormones such as auxin, and presumably via modulating local auxin biosynthesis and/or intercellular auxin transport within anther tissues, thus triggering anther development.

\section{Discussion}

Using an artificial microRNA approach, we generated constitutive knockdown lines targeting the gene RUS4, which belongs to the DUF647 family that is conserved in eukaryotic organisms, but has no known biological function. We present the first evidence for the important role of RUS4 gene in Arabidopsis anther dehiscence by affecting secondary wall thickening in the endothecium.

\section{RUS4 is involved in the process of anther dehiscence in Arabidopsis}

The down-regulation of RUS4 gene in amiR-RUS4 plants resulted in high levels of male sterility due to a defect in anther dehiscence. Examination of anther transverse sections indicated that the amiR-RUS4 plants underwent relatively normal anther development during the early stages, including the 
specification of cells, differentiation of tissues, and degeneration of the tapetum (Fig. 4A, B). However, striking defects occurred during the later stages of anther development, in which normal endothecium secondary wall thickenings failed to form (Fig. 4D, F, H; Fig. 5B, E). The amiR-RUS4 anther indehiscence phenotype resembles that of myb26 (Yang et al. 2007) and the nst1nst2 double mutant (Mitsuda et al. 2005); both these mutants show associated changes in expression of genes encoding cellulose synthase catalytic subunits (CESAs), resulting in an absence of endothecium secondary thickening and failure of anther dehiscence. A similar effect was seen in the amiR-RUS4 plants, which showed a correlation between levels of NST1 and NST2 transcripts and the expression of the down-stream CESA/IRX genes (Fig. 6). However, the expression MYB26, a known direct regulator of NST1 and NST2, was not reduced in amiR-RUS4 flower buds. Rather, MYB26 showed enhanced and prolonged expression (Supplementary Fig. S4A), suggesting the impact of RUS4 on endothecium secondary thickening may be independent of MYB26. This hypothesis is reinforced by the observation that RUS4 expression did not change in the myb26 mutant (Supplementary Fig. S4B). Furthermore, overexpression of RUS4 cannot suppress the myb26 mutant phenotype (Supplementary Fig. S4D). Thus, RUS4 may represent a new player in control of anther dehiscence, which is not directly associated with the regulation of endothecium secondary thickening by MYB26.

RUS4 affects endothecium secondary thickening formation by indirect modulation of the expression of secondary thickening related genes

Our study showed that a number of genes linked to secondary thickening exhibited significant changes in expression in amiR-RUS4 plants. Importantly, transcript levels of NST1 and NST2 genes were dramatically reduced at floral stage 10 to 11 (Fig. 6A, B), just before endothecium lignification, in the amiR-RUS4 plants compared with the wild-type. In agreement with the reduced level of secondary 
thickening in the endothecium, the transcript levels of the secondary cell wall thickening-associated MYB transcription factor genes, such as MYB103 and MYB85 (Zhong et al. 2008), were also significantly reduced in amiR-RUS4 flower buds, especially at flower stage 11 when endothecium lignification occurs (Fig. 6C, D). Furthermore, several cellulose synthase (CESA) genes were markedly decreased at the transcript levels in the amiR-RUS4 buds (Fig. 6E-G). In contrast, overexpression of RUS4 led to increased NST1 and NST2, as well as downstream MYB103 and MYB85 transcript levels (Fig. 7B-E), and enhanced lignified endothecium secondary thickening in the anthers (Fig. 5C, F; Supplementary Fig. S5B-D, F-H), and also increased secondary thickening in the filaments (Fig. 5I; Supplementary Fig. S5J-L). Therefore, it appears that RUS4 may promote anther endothecium secondary thickening via affecting the expression of the secondary cell wall thickening-associated transcription factor genes; this in turn allows regulation of the secondary wall biosynthetic program.

Proteomic studies suggest that RUS4 is localized to the chloroplast (Kaundal et al., 2010). We confirmed this using the RUS4-GFP lines. The chloroplast-localization of RUS4 indicates that it affects the expression of secondary wall transcription factors would be an indirect effect rather than a direct regulation; this is possibly the consequences of the abnormal development of the endothecium. Alternatively, RUS4 might also have a role in auxin transport, just like RUS1 and RUS2, and failure of endothecium secondary thickening in amiR-RUS4 anthers could be brought about by the altered auxin levels in the anther tissues. Altered auxin accumulation would trigger rapid transcriptional responses, thus influencing the expression of specific set of genes, such as NST1 and NST2. This in turn allows regulation of secondary thickening in the endothecium (Fig. 9). There are already excellent demonstrations that the distribution of correct amounts of auxin in anther tissues plays an important role in late stamen development in Arabidopsis (Cecchetti et al. 2008; Cecchetti et al. 2017). In cotton, 
abnormal accumulation of auxin also resulted in anther indehiscence due to reorientation of the cytoskeleton and alterations in secondary wall thickening in anther endothecium (Yasuor et al. 2006). Whether RUS4 is involved in modulation of the auxin transport and/or homeostasis is an interesting direction for future investigation.

\section{The DUF647 family genes and plant development}

The Arabidopsis genome encodes six DUF647 family members. Two of these, RUS1/WXR3 and RUS2/WXR1, have been identified as loss-of-function mutations with defects in auxin response and UV-B light sensing (Tong et al. 2008, Leasure et al. 2009, Ge et al. 2010, Yu et al. 2013). Both of the mutants have very similar phenotypes with defects in root development, polar auxin transport, and response to UV-B. The rus1rus2 double mutant plants exhibit more severe defects in auxin transport, as well as root and shoot growth (Leasure et al. 2009, Yu et al. 2013), suggesting that RUS1 and RUS2 have overlapping function in plant vegetative growth, but are not likely to play the principle role in reproductive development in Arabidopsis. RUS4 is therefore the first DUF647 family gene to be associated with plant reproduction development and to play an important role in secondary wall thickening in anther endothecium. It is reasonable that RUS1, RUS2 and RUS4 have distinct functions in plant growth and development. Firstly, two-protein BLAST alignments show that the identity between RUS4 and RUS1, RUS2 is low at $19.6 \%$ and $21.2 \%$, respectively (EMBOSS Needle, Rice et al. 2000, Supplementary Table S2). Secondly, promoter-GUS fusions indicate that the RUS1/WXR3 and RUS2/WXR1 promoter is active in the cotyledons, roots, and hypocotyls, and particularly strong GUS staining was observed in the leaf veins, root vascular tissues, root tip and lateral root primordial (Ge et al. 2010, Yu et al. 2013), suggesting the primary function of RUS1/WXR3 and RUS2/WXR1 is in lateral root initiation. In contrast, RUS4pro:GUS fusion indicated that RUS4 is strongly expressed in 
the anthers, consistent with its role in anther development (Fig. 2). Even though the RUS4 T-DNA knockout mutant rus4-2 showed no obvious developmental defects, the complementation of the amiRRUS4 fertility phenotype with RUS4 cDNA (Fig. 1B, E) confirmed that the described anther defects are due to the loss of RUS4 function. The discrepancy of the phenotype between the RUS4 knockout mutant rus 4-2 and the RUS4-amiRNA knockdown line could be explained by a genetic compensation induced by RUS4 knockout mutation. Such compensation has been observed in zebrafish egfl7 and vegfaa mutants in which a set of proteins and genes were upregulated (Rossi et al. 2015). Rossi et al. (2015) suggested that a compensatory network can be activated to buffer against deleterious mutations such as null mutation but not after transcriptional or translational knockdown mutations. Our qPCR analysis of the DUF647 family genes showed that RUS6 expression was slightly upregulated in rus4-2 mutant (Supplementary Figure S2D), and phylogenetic analysis showed that RUS6 has the closest relationship with RUS4 (Ge et al., 2010), suggesting a possible role of RUS6 in compensation for the loss of RUS4 function. However, other DUF647 family members such as RUS5 has displayed similar expression patterns to RUS4 in floral organs, as illustrated in Arabidopsis eFP Brower at http://bar.utoronto.ca (winter et al. 2007), implying that RUS5 may also have a role in reproductive development. It will be interesting, in the future, to determine which member(s) of the DUF647 gene family is acting redundantly with RUS4, and whether a similar function is offered by additional proteins active in the anther.

\section{Materials and Methods}

Plant materials and growth conditions

Arabidopsis (Arabidopsis thaliana) wild-type and amiR-RUS4 plants used in this study were of Columbia (Col-0) ecotype. Seed stocks of rus4-1 (At2g23470; GK-345D06) and rus4-2 (At2g23470; 
GK-447F02), both in Col-0 background, were obtained from the Arabidopsis Biological Resource Center (ABRC, Ohio State University). The myb26 mutant SALK_112372 in Col-0 background was used as previously described by Yang et al. (2007). Seeds were sown on Levington-M3 (Scotts):vermiculite (3:1; w/w) compost mix and grown under white light with $16 \mathrm{~h}$ light $/ 8 \mathrm{~h}$ dark cycles at $22 / 18^{\circ} \mathrm{C}$ (day/night). For seedling experiments, seeds were surface sterilized in $30 \%(\mathrm{v} / \mathrm{v})$ bleach, plated on half-strength Murashige and Skoog (MS) medium with 1\%(w/v) sucrose and 1\%(w/v) agar, pH 5.9, and incubated at $4{ }^{\circ} \mathrm{C}$ for 2 days before transferring to the growth room. Seedlings were grown on vertical plates at $22^{\circ} \mathrm{C}$ under long-day conditions (16 h of light/8 h of dark).

Generation of transgenic Arabidopsis plants expressing amiRNA targeting RUS4

For generating the amiRNA construct to knock down the expressions of RUS4, we designed an optimal amiRNA sequence using the WMD (Web MicroRNA Designer, (WMD version 3; http://wmd.weigelworld.org/) platform. The designed target 21-mer amiRNA sequence (5 ' TGACAAATAACGATGCCCCAT-3') was synthesized by the overlapping PCR according to the method described in Schwab et al. (2006) and Ossowski et al. (2008). Primers are listed in Supplementary Table S1. The amplified amiRNA fragment was then introduced into the pCHF3 vector to generate RUS4-amiRNA/pCHF3. The resulting plasmid that harbor RUS4-amiRNA driven by Cauliflower Mosaic Virus 35S (CaMV 35S) promoter was then introduced into Agrobacterium tumefaciens strain AGL1, and subsequently transformed into Arabidopsis (Col-0) plants by the floral dip method (Clough and Bent 1998). Transgenic T1 plants were identified by selection for kanamycin resistance, and surviving plants were genotyped to confirm the presence of the transgene. The homozygous T3 seeds and their progeny were used for further experiments. 
RUS4 overexpression lines

A 1,794 bp DNA fragment encoding RUS4 was amplified from wild-type Col-0 buds cDNA using Phusion DNA Polymerase (Thermo Fisher Scientific) using primers spanning the full-length cDNA and including a stop codon (Supplementary Table S1: RUS4-F1 and RUS4-R1). The fragment was cloned into a Gateway-compatible entry vector (pCR8/GW/TOPO; Invitrogen) and confirmed by sequencing. The RUS4 cDNA was recombined into a Gateway-compatible destination binary vector pGWB5 using LR Clonase II enzyme mix (Invitrogen) according to the manufacturer's instructions. The construct was transformed into Agrobacterium tumefaciens C58 pGV3850 and then introduced into Arabidopsis wild-type Col-0, amiR-RUS4 background and also heterozygous myb26 SALK plants by floral dipping (Clough and Bent, 1998). The T1 plants were selected on MS plates with $50 \mathrm{mg} \mathrm{L}^{-1}$ hygromycin and PCR tested for the transgene. The selected homozygous lines of 35Spro:RUS4 in the heterozygous myb26/MYB26 background were then subsequently genotyped to identify overexpression of RUS4 lines in the homozygous myb26 background.

\section{RUS4pro:GUS expression assay}

A 1,957-bp region of genomic DNA upstream of the RUS4 gene was PCR amplified and cloned into pCR8/GW/TOPO (Invitrogen, http://www.invitrogen.com/) to create pCR/TOPO-RUS4pro. The promoter region was recombined into the Gateway-compatible destination binary vector pGWB3 using LR clonase II enzyme mix (Invitrogen) according to the manufacturer's instruction. The RUS4pro:GUS construct was then transferred into Agrobacterium (C58 pGV3850) by electroporation and transformed into Arabidopsis wild-type (Col-0 ecotype) by floral dip method. T1 seeds were grown on MS plates with $50 \mathrm{mg} \mathrm{L}^{-1}$ hygromycin, and surviving plants were then genotyped to confirm the presence of the transgene. The transgenic plants were grown to maturity and allowed to self-pollinate. 
GUS staining was performed according to Jefferson et al. (1987) and Lindsey et al. (2000) with slight modifications. Arabidopsis seedlings, harvested after 7 days of growth on vertical agar plates, and flower buds of transgenic plants were collected and placed in GUS staining solution (50 mM phosphate buffer, pH7.0, 0.1\% Triton X-100, $0.05 \mathrm{mM}$ potassium ferricyanide, $0.05 \mathrm{mM}$ potassium ferrocyanide and 1 $\mathrm{mM}$ 5-bromo-4-chloro-3-indolyl- $\beta$-glucuronide) to visualize GUS activity. Samples were placed in a $37^{\circ} \mathrm{C}$ incubator for 48 hours. Tissues were first clarified by acidified methanol $\left(38 \mathrm{ml} \mathrm{H}_{2} \mathrm{O}, 10 \mathrm{ml}\right.$ Methanol, $2 \mathrm{ml} \mathrm{HCI}$ ) at $55^{\circ} \mathrm{C}$ for $20 \mathrm{~min}$, then cleared with $7 \% \mathrm{NaOH}$ in $60 \%$ ethanol at room temperature for $20 \mathrm{~min}$, followed by an ethanol series clarification, seedlings and dissected flowers were then observed and photographed with a light microscope.

\section{Subcellular Localization of RUS4 in Planta}

The RUS4 CDS without the stop codon was PCR amplified from cDNA using primers RUS4-F2 and RUS4-R2, and the resulting PCR product was separated on a 1\% agarose gel and purified using a QIAquick gel extraction kit. The blunt-end PCR product was cloned into the Gateway entry vector pENTR-D-TOPO vector using pENTR directional TOPO cloning kit (Invitrogen). The entry clone was confirmed by DNA sequencing, and then sub-cloned into the destination vector pMDC83 using the Gateway LR reaction kit (Invitrogen). This produced a C-terminal fusion with GFP driven by a double 35S promoter. The 35Spro:RUS4-GFP construct was stably transformed into Arabidopsis amiR-RUS4 and Col-0 genetic backgrounds using the Agrobacterium-mediated floral dip method (Clough and Bent 1998). To visualize GFP and chlorophyll in young leaves and hypocotyls, samples were observed under a Zeiss Observer A1 microscope using excitation band-pass (BP) 450-490 nm, BS $510 \mathrm{~nm}$ filters and emission BP 515-555 nm. Images obtained at the two spectral settings were overlaid. 


\section{Light microscopy}

Observation of anther development was performed using anther transverse sections as described by Li et al. (2006). Floral buds at various developmental stages were collected and fixed with FAA, which contained $5 \%(\mathrm{v} / \mathrm{v})$ formaldehyde $(37 \%), 5 \%(\mathrm{v} / \mathrm{v})$ acetic acid, and $63 \%(\mathrm{v} / \mathrm{v})$ ethanol, and dehydrated in an ethanol series. The samples were embedded in Technovit 7100 resin (Hereaus Kulzer), polymerized at $45^{\circ} \mathrm{C}$. Transverse sections of $3 \mu \mathrm{m}$ were cut using a Microtome (Leica RM2265, Germany) stained with $1 \%$ toluidine blue $\mathrm{O}$ and photographed using a Nikon E600 microscope and a Nikon DXM1200 digital camera. Anther stages were referred as described by Sanders et al. (1999).

\section{Scanning electron microscopy}

Wild-type and amiR-RUS4 anthers and pollen grains were collected from flowers during each of the stage 13 of anther development. Material was fixed in FAA ( $5 \%(\mathrm{v} / \mathrm{v})$ formalin $(37-40 \%), 5 \%(\mathrm{v} / \mathrm{v})$ acetic acid, and 50\% (v/v) ethanol) for $24 \mathrm{~h}$, dehydrated with a graded ethanol series $(30,50,70,80,90$, 95 and $100 \%$ ), each for $20 \mathrm{~min}$, air dried in silica, coated with gold particles, and observed using a JSM-35C scanning electron microscope at an accelerating voltage of $25 \mathrm{kV}$.

\section{Confocal microscopy of the cell wall}

Anthers stained with ethidium bromide/acridine orange were visualized by confocal microscopy according to Yang et al. (2007). The ethidium bromide stains lignified cells (red fluorescence; $514 \mathrm{~nm}$ excitation; emission collection at 580-620 nm), and the acridine orange stains non-lignified walls (green fluorescence; $458 \mathrm{~nm}$ excitation; emission collection at 480-510 nm).

\section{RNA extraction and quantitative RT-PCR}

Total RNA was extracted from seedlings, rosette leaves, stems, flowers, flower buds and siliques using 
the RNeasy Plant Mini Kit (Qiagen). Seedlings were harvested after 14 days of growth on vertical agar plates. Various tissues were harvested from soil-grown plants when the primary inflorescence was fully elongated. Closed floral buds were harvested at stage $\leq 12$, and flower buds at various developmental stages $<9,10,11$ and 12 were also collected separately. Flowers were harvested at stage 13 and siliques 4 days past stage 14. Yield was quantified using a Thermo Scientific NanoDrop 2000. A total of $5 \mu \mathrm{g}$ of RNA was used for complementary DNA synthesis through the SuperScript III First-Strand Synthesis Kit (Invitrogen). Quantitative RT-PCR (qRT-PCR) analyses were performed on a LightCycler 480 II instrument (Roche) and ABI StepOne Plus Real-Time System (Life Technologies), using the Maxima SYBR Green/ROX qPCR MasterMix (Fermentas). The primers are listed in Supplementary Table S1. All samples were run in triplicate. Data were normalized either to the reference gene PROTEIN PHOSPHATASE2A-2 (PP2A-2) or ACTIN2.

\section{Statistical analysis}

Student's $t$ tests were used to evaluate statistical significance. All the statistical analyses were performed using Statistical Product and Service Solutions (SPSS) 17.0 software.

Accession numbers

The Arabidopsis Genome Initiative locus identifiers for genes mentioned in this article are as follows: RUS4 (At2g23470), MYB26 (At3g13890), MYB85 (At4g22680), MYB103 (At1g63910), NST1 (At2g46770), NST2 (At3g61910), IRX1 (At4g18780), IRX3 (At5g17420), IRX5 (At5g44030) and IRX8 (At5g54690).

\section{Funding}

This work was supported by grants from the National Natural Science Foundation of China (31170273 
to S.-Q. Z.), the International Cooperation Project of Shanxi Science and Technology (2009081005 to S.-Q. Z.), Shanxi Scholarship Council of China (2016-013 to S.-Q. Z.), Taiyuan Scientific Celebrity Special (11014902 to S.-Q. Z.) and the Biotechnology and Biological Sciences Research Council (BBSRC) (BB/J001295/1 to Z.W.).

\section{Disclosures}

The authors have no conflicts of interest to declare.

\section{Acknowledgements}

We thank the Arabidopsis Biological Resource Center (The Ohio State University) for providing the RUS4 T-DNA seeds. We are grateful to Prof. Mark Estelle (Cell and Developmental Biology, University of California San Diego, California, USA) for supporting the creation of artificial microRNA plants. We thank Dr. Jie Xu and MSc. Xu Chen (School of Life Sciences and Biotechnology, Shanghai Jiao Tong University, Shanghai, China) for technical assistance with anther transverse sections analysis, Dr. Nobutaka Mitsuda (Gene Function Research Center, National Institute of Advanced Industrial Science and Technology, Tsukuba, Japan) for kindly providing the pro35S:NST1 and pro35S:NST2 constructs, and Dr. Yuxin Hu (Institute of Botany, Chinese Academy of Sciences, Beijing, China) for the pMDC83 vector.

\section{References}

Bonner, L. and Dickinson, H. (1989) Anther dehiscence in Lycopersicon esculentum Mill. New Phytol. 113: $97-115$.

Bowman, J.L., Smyth, D.R, and Meyerowitz, E.M. (1991) Genetic interactions among floral homeotic genes of Arabidopsis. Development 112:1-20. 
Brown, D.M., Zeef, L.A., Ellis, J., Goodacre. R., and Turner, S.R. (2005) Identification of novel genes in Arabidopsis involved in secondary cell wall formation using expression profiling and reverse genetics. Plant Cell 17: 2281-2295.

Cecchetti, V., Altamura, M.M., Falasca, G., Costantino, P., Cardarelli, M. (2008) Auxin regulates Arabidopsis anther dehiscence, pollen maturation, and filament elongation. Plant Cell 20: 17601774.

Cecchetti, V., Celebrin, D., Napoli, N., Ghelli, R., Brunetti, P., Costantino, P., et al. (2017) An auxin maximum in the middle layer controls stamen development and pollen maturation in Arabidopsis. New Phytol 213: 1194-1207.

Clough, S.J. and Bent, A.F. (1998) Floral dip: A simplified method for Agrobacterium-mediated transformation of Arabidopsis thaliana. Plant J. 16: 735-743.

Cui, X., Wang, Q., Yin, W., Xu, H., Wilson, Z.A., Wei, C., et al. (2012) PMRD: a curated database for genes and mutants involved in plant male reproduction. BMC Plant Biol. 12: 215-224.

Dobritsa, A.A., Geanconteri, A., Shrestha, J., Carlson, A., Kooyers, N., Coerper, D., et al. (2011) A large-scale genetic screen in Arabidopsis to identify genes involved in pollen exine production. Plant physiol. 157: 947-970.

Ge, L., Peer, W., Robert, S., Swarup, R., Ye, S., Prigge, M., et al. (2010) Arabidopsis ROOT UVB SENSITIVE2/WEAKAUXINRESPONSE1 is required for polar auxin transport. Plant Cell 22: 17491761.

Goldberg, R.B., Beals, T.P., and Sanders, P.M. (1993) Anther development: Basic principles and practical applications. Plant Cell 5: 1217-1229. 
Hao, Z., Avci, U., Tan, L., Zhu, X., Glushka. J., Pattathil, S. et al. (2014) Loss of Arabidopsis

GAUT12/IRX8 causes anther indehiscence and leads to reduced G lignin associated with altered matrix polysaccharide deposition. Front. Plant Sci. 5: 357.

Jakobsen, M.K., Poulsen, L.R., Schulz, A., Fleurat-Lessard, P., Møller, A., Husted, S. et al. (2005)

Pollen development and fertilization in Arabidopsis is dependent on the MALE GAMETOGENESIS

IMPAIRED ANTHERS gene encoding a Type V P-type ATPase. Genes Dev. 19: 2757-2769.

Jefferson, R.A., Kavanagh, T.A., and Bevan, M. W. (1987) GUS fusions: beta-glucoronidase as a sensitive and versatile gene fusion marker in higher plants. EMBO Journal. 13: 3901-3907.

Jung, K.W., Kim Y.Y., Yoo K.S., Ok, S.H., Cui, M.H., Jeong, B-C. et al. (2013) A Cystathionine-bSynthase Domain-Containing Protein, CBSX2, regulates endothecial secondary cell wall thickening in anther development. Plant Cell Physiol. 54: 195-208.

Kaundal, R., Saini, R., Zhao, P.X. (2010) Combining machine learning and homology-based approaches to accurately predict subcellular localization in Arabidopsis. Plant Physiol 154: 36-54.

Keijzer, C.K. (1987) The processes of anther dehiscence and pollen dispersal I. The opening mechanism of longitudinally dehiscence anthers. New Phytol. 105: 487-498.

Keijzer, C.J., Leferink-Ten Klooster HB, Reinders MC. (1996) The mechanics of the grass flower: anther dehiscence and pollen shedding in maize. Ann. Bot. 78: 15-21.

Kim, Y.Y., Jung, K.W., Jeung, J.U. and Shin, J.S. (2012) A novel F-box protein represses endothecial secondary wall thickening for anther dehiscence in Arabidopsis thaliana. J. Plant Physiol. 169: 212216.

Leasure, C.D., Tong, H., Yuen, G., Hou, X., Sun, X., and He, Z.H. (2009) ROOT UV-B SENSITIVE2 acts with ROOTUV-B SENSITIVE1 in a root ultraviolet B-sensing pathway. Plant Physiol. 150: 
1902-1915.

Li, N., Zhang D.S., Liu H.S. Yin C.S., Li X.X., Liang W.Q. et al. (2006) The rice tapetum degeneration retardation gene is required for tapetum degradation and anther development. Plant Cell 18: 29993014.

Lindsey, K. and Wei, W. (2000) Tissue culture, transformation, and transient gene expression in Arabidopsis. In Arabidopsis: A Practical Approach (Wilson, Z.A. ed.). Oxford University Press. pp125-141.

Matsui T, Omasa K, Horie T. (1999) Mechanism of anther dehiscence in rice (Oryza sativa L.). Annals of Botany 84: 501-506.

Mitsuda, N., Seki, M., Shinozaki, K., Ohme-Takagi, M. (2005) The NAC transcription factors NST1 and NST2 of Arabidopsis regulate secondary wall thickenings and are required for anther dehiscence. Plant Cell 17: 2993-3006.

Nelson, M.R., Band, L.R., Dyson, R.J., Lessinnes, T., Wells, D.M., Yang, C. et al. (2012) A biomechanical model of anther opening reveals the roles of dehydration and secondary thickening. New Phytol 196: 1030-1037.

Öhman, D., Demedts B, Kumar M, Gerber, L., Gorzsás, A., Goeminne, G. et al. (2013) MYB103 is required for FERULATE-5-HYDROXYLASE expression and syringyl lignin biosynthesis in Arabidopsis stems. Plant J. 73: 63-76.

Ossowski, S., Schwab, R. and Weigel, D. (2008) Gene silencing in plants using artificial microRNAs and other small RNAs. Plant J. 53: 674-690.

Persson, S., Caffall, K.H., Freshour, G., Hilley, M.T., Bauer, S., Poindexter, P. et al. (2007) The Arabidopsis irregular xylem 8 mutant is deficient in glucuronoxylan and homogalacturonan, which 
are essential for secondary cell wall integrity. Plant Cell 19: 237-255.

Quilichini, T.D., Grienenberger, E. and Douglas, C.J. (2015) The biosynthesis, composition and assembly of the outer pollen wall: A tough case to crack. Phytochemistry 113: 170-182.

Rice, P., Longden, I., and Bleasby, A. (2000) EMBOSS: the European Molecular Biology Open Software Suite. Trends Genet. 16: 276-277.

Rossi, A., Kontarakis, Z., Gerri, C., Nolte, H., Holper, S., Krüger, M., et al. (2015) Genetic compensation induced by deleterious mutations but not gene knockdowns. Nature 524: 230-233.

Sanders, P.M., Bui, A.Q., Le, B.H. and Goldberg, R.B. (2005) Differentiation and degeneration of cells that play a major role in tobacco anther dehiscence. Sex. Plant Reprod. 17: 219-241.

Sanders, P.M., Bui, A.Q., Weterings, K., McIntire, K.N., Hsu, Y.C., Lee, P.Y. et al. (1999) Anther developmental defects in Arabidopsis thaliana male-sterile mutants. Sex. Plant Reprod. 11: 297-322.

Schwab, R., Ossowski, S., Riester, M., Warthmann, N., and Weigel, D. (2006) Highly specific gene silencing by artificial microRNAs in Arabidopsis. Plant Cell 18: 1121-1133.

Scott, R.J., Spielman, M., and Dickinson, H.G. (2004) Stamen structure and function. Plant Cell 16: S46-S60.

Smyth, D.R., Bowman, J.L. and Meyerowitz, E.M. (1990) Early flower development in Arabidopsis. Plant Cell 2: 755-767.

Taylor NG, Laurie S, Turner SR. 2000. Multiple cellulose synthase catalytic subunits are required for cellulose synthesis in Arabidopsis. Plant Cell 12: 2529-2539.

Taylor, N.G., Howells, R.M., Huttly, A.K., Vickers, K. and Turner, S.R. (2003) Interactions among three distinct CesA proteins essential for cellulose synthesis. Proc. Natl. Acad. Sci. USA 100: 14501455. 
Taylor, N.G., Scheible, W.R., Cutler, S., Somerville, C.R. and Turner, S.R. (1999) The irregular xylem 3 locus of Arabidopsis encodes a cellulose synthase required for secondary cell wall synthesis. Plant Cell 11: 769-780.

Tong, H., Leasure, C.D., Hou, X., Yuen, G., Briggs, W., and He, Z.H. (2008) Role of root UV-B sensing in Arabidopsis early seedling development. Proc. Natl. Acad. Sci. USA 105: 21039-21044.

Wilson, Z.A., and Zhang, D.B. (2009) From Arabidopsis to rice: pathways in pollen development. $J$. Exp. Bot. 60: 1479-1492.

Wilson, Z.A., Song, J., Taylor, B., and Yang, C. (2011) The final split: the regulation of anther dehiscence. J. Exp. Bot. 62: 633-1649.

Winter, D., Vinegar, B., Nahal, H., Ammar, R., Wilson, G.V., Provart, N.J. (2007) An "Electronic Fluorescent Pictograph" browser for exploring and analyzing large-scale biological data sets. PLoS One. 2(8): e718.

Yang, C., Song, J., Ferguson, A., Klisch, D., Simpson, K., Mo, R. et al. (2017) Transcription factor MYB26 is key to spatial specificity in anther secondary thickening formation. Plant Physiol. 175: $333-350$.

Yang, C., Xu, Z., Song, J., Conner, K., Vizcay Barrena, G., and Wilson, Z.A. (2007) Arabidopsis MYB26/MALE STERILE35 regulates secondary thickening in the endothecium and is essential for anther dehiscence. Plant Cell 19: 534-548.

Yasuor, H., Abu-Abied, M., Belausov, E., Madmony, A., Sadot, E., Riov, J. et al. (2006) Glyphosateinduced anther indehiscence in cotton is partially temperature dependent and involves cytoskeleton and secondary wall modifications and auxin accumulation. Plant Physiol. 141: 1306-1315.

Yu, H., Karampelias, M., Robert, S., Peer, WA., Swarup, R., Ye, S. et al. (2013) ROOT 
ULTRAVIOLET B-SENSITIVE1/WEAK AUXIN RESPONSE3 is essential for polar auxin transport in Arabidopsis. Plant physiol. 162: 965-976.

Zhang, D.B. and Yang, L. (2014) Specification of tapetum and microsporocyte cells within the anther. Curr. Opin. Plant Biol. 17: 49-55.

Zhong, R., Lee, C., Zhou, J., McCarthy, R.L., and Ye, Z.-H. (2008) A battery of transcription factors involved in the regulation of secondary cell wall biosynthesis in Arabidopsis. Plant Cell 20: 27632782.

Zhong, R., and Ye Z.-H. (2015) Secondary cell walls: biosynthesis, patterned deposition and transcriptional regulation. Plant Cell Physiol. 56(2): 195-214.

Zhong R, Cui D and Ye Z.-H. (2018) Secondary cell wall biosynthesis. New Phytol. doi: 10.1111/nph.15537.

\section{Figure legends}

Fig. 1 Analysis of RUS4-amiRNA overexpressor.

(A) Alignment of amiRNA to RUS4. The position of amiRNA ('subject') binding relative to the translated region of RUS4 is shown. (B) qRT-PCR of RUS4 transcript levels in floral buds of wild-type, amiR-RUS4 and CaMV 35S:RUS4/amiR-RUS4 plants. Values are the means $\pm \mathrm{SD}(\mathrm{n}=3)$. ACTIN2 was used as a reference gene. (C) Six-week-old plants of wild-type and the amiR-RUS4 line. The amiRRUS4 plants resemble wild-type, except that the siliques are small, undeveloped and devoid of seeds (arrow). (D) Siliques from reciprocal crosses between wild-type and the amiR-RUS4 line demonstrate that the male fertility of amiR-RUS4 plants is compromised. (E) The primary inflorescences from wildtype plants, the amiR-RUS4 plants and the amiR-RUS4 plants expressing CaMV 35S:RUS4 transgene. The amiR-RUS4 plants containing the CaMV 35S:RUS4 showed rescue with elongated siliques 
(arrow). (F) Seed yield of wild-type and the amiR-RUS4 line. Data represent the mean \pm SD of 12

plants. Scale bars $=1 \mathrm{~cm}$ in D.

Fig. 2 RUS4 expression pattern. (A) Expression of RUS4 in various tissues analyzed by qRT-PCR. Flowers were harvested at stage 13, floral buds at stage 12 and younger, and siliques 4 days after stage 14 (Smyth et al., 1990). Stems were harvested from soil-grown plants, when the primary inflorescence was fully elongated. Seedlings were harvested after 14 days of growth on vertical agar plates. Vegetative rosette leaves were harvested from 26-days-old plants. ACTIN2 was used as a normalization control. Bars represent mean \pm SD of three replicates. (B-F) GUS staining of flowers at different developmental stages of RUS4pro:GUS transgenic plants. (B) Early stage buds (< stage 9) showed no GUS staining. (C) Weak GUS signal was visible in stage 9 buds (arrow), strong GUS staining was visualized in older anthers at stage 10 (arrowhead). (D, E) Intense GUS staining was observed in the anthers at stages 11 and 12. (F) GUS staining remained relatively strong in anthers at stage 13. Released pollen grains also showed GUS staining (arrow). (G) The mature, shed pollen grains showed GUS staining. (H) Anther squashed from stage 10 floral bud showed GUS activity in the tapetum (T). (I) Anther dissected from stage 11 flower buds showed strong GUS activity in the endothecium (En), pollen grains (PG), and weak GUS activity in the vascular bundle (VB) of the filament. Scale bars $=500 \mu \mathrm{m}$ in B-F; $20 \mu \mathrm{m}$ in G; 50 $\mu \mathrm{m}$ in H, I. Flower development stages are defined according to Smyth et al. (1990) and Bowman et al. (1991).

Fig. 3 Analysis of the amiR-RUS4 line flower, anther and stigma (A, B) Flowers immediately after opening.

Wild-type Col-0 flower showing dehiscent anthers (A), amiR-RUS4 flower is of normal size, the 
stamen filaments elongate properly, but anthers fail to dehisce (B). (C, D) Pollen release test showing released pollen grains from a wild-type anther $(\mathrm{C})$, no pollen release was observed from the amiRRUS4 anther (D). (E, F) DIC microscopy of wild-type and amiR-RUS4 stigmas excised from an open flower. Numerous pollen grains were attached to the stigma of wild-type flower (E, arrow), but no pollen grains were visible on the stigma surface of the amiR-RUS4 flower (F). (G-H) SEM analysis of wild-type and amiR-RUS4 anthers. Anther at stage 13 of wild-type (G) and amiR-RUS4 (H, I). Bars = $50 \mu \mathrm{m}$.

Fig. 4 Comparison of wild-type and amiR-RUS4 anther development.

Semithin anther sections were stained with toluidine blue O, with two locules shown in each except (J); wild-type (A, C, E, G and I) and amiR-RUS4 (B, D, F, H and J). (A, B) At stage 10, uninucleate microspores were formed, tapetum degeneration initiated in both the wild-type and amiR-RUS4 mutant anthers. (C, D) At stage 11, endothecium cell were expanded, with fibrous bands of thickening present in the endothecium and connective in the wild-type anther (C), whereas although the endothecium layer could be seen in the amiR-RUS4 anther, only limited expansion and fibrous bands were observed (D). Pollen grains formed exine in both wild-type and the amiR-RUS4 anthers. (E, F) At stage 12, the stomium was broken down in both wild-type and amiR-RUS4 anthers. (G, H) At stage 13, stomium breakage and endothecium thickening resulted in anther dehiscence and pollen release in wild-type $(G)$, a lack of endothecium secondary thickening and associated failure of stomium breakage in the amiR-RUS4 anthers resulted in indehiscence (H). (I, J) At stage 14, anther shrinkage, and cell distortion was seen in both the wild-type and the amiR-RUS4 line. However amiR-RUS4 anthers still did not dehisce (J). C, connective; E, epidermis; En, endothecium; Fb, fibrous bands; PG, pollen grain; Sm, septum; St, stomium; StR, stomium region; $\mathrm{T}$, tapetum; $\mathrm{V}$, vascular region. Bars $=50 \mu \mathrm{m}$. 
Fig. 5 Stamens stained for secondary thickening with acridine orange/ethidium bromide and visualized by confocal microscopy.

(A, D) wild-type anther showing lignified endothecium layer; (B, E) amiR-RUS4 anther showing only few lignified endothecium cells; (C, F) a representative RUS4 overexpression line showing high levels of secondary thickening in the endothecium (C and F). (G-I) the filaments of wild-type (G), amiRRUS4 (H) and one RUS4-OE line (I). The RUS4-OE line showing increased secondary thickening in the filament (I). Scale bars represent $50 \mu \mathrm{m}$ in A to C, $10 \mu \mathrm{m}$ in D to I.

Fig. 6 Expression of genes related to the thickening of the secondary cell wall in amiR-RUS4 flower buds.

Quantitative RT-PCR analysis of (A) NST1, (B) NST2, (C) MYB103, (D) MYB85, (E) CESA4, (F) CESA7, (G) CESA8 and (H) GAUT12 transcript levels in wild-type and amiR-RUS4 flower buds at various developmental stages: up to 9 (indicated as $\leq 9$ ), 10, 11 and 12 (Smyth et al., 1990). The relative levels of each gene in the youngest buds (stage $\leq 9$ ) of the wild type was set at $1 . P P 2 A$ transcript were used as a normalization control in (A, B) and (E-H); ACTIN2 transcript were used as a normalization control in (C, D). The data represent the average value $\pm \mathrm{SD}(\mathrm{n}=3)$.

Fig. 7 Expression of genes related to the secondary cell wall thickening in flower buds from the RUS4 overexpression plants.

(A) qRT-PCR analysis of RUS4 expression in the unopened flower buds of wild-type and three independent RUS4 overexpression lines. (B, C) Relative expression levels of NST1 (B) and NST2 (C) in flower buds at stages 10 and 11 from wild-type and a representative RUS4 overexpression line (OE-8) measured by qRT-PCR. (D, E) Relative expression levels of MYB103 (D) and MYB85 (C) in wild-type 
and the RUS4 overexpression line (OE-8) flower buds at developmental stages: up to 9 (indicated as $\leq$ 9), 10, 11 and 12 measured by qRT-PCR. ACTIN2 was used as a normalization control. The data represent the average value $\pm \mathrm{SD}(\mathrm{n}=3)$. Asterisks indicate a significant difference from the wild-type value; $* P<0.05, * * P<0.01$, Student $t$-test.

Fig. 8 RUS4:GFP localizes to the chloroplast.

Subcellular localization of RUS4-GFP in Arabidopsis leaves (A to C) and hypocotyls (D to F) in homozygous transgenic lines expressing the fusion protein from the $35 \mathrm{~S}$ promoter. (A, D), Fluorescence images of the Arabidopsis amiR-RUS4 (A) and wild-type Col-0 (D) expressing 35Spro:RUS4-GFP; (B, E) autofluorescence of the chloroplast; (C, F) an overlay of image (A) and (B), and (D) and (E), respectively. RUS4-GFP co-localizes with autofluorescence of the chloroplasts in Arabidopsis 35Spro:RUS4-GFP leaves (C) and hypocotyls (F). Bars $=20 \mu \mathrm{m}$.

Fig. 9 Speculative model for the action of RUS4.

The plastid-localized RUS4, possibly together with other proteins, may play a role in the regulation of auxin transport, with consequent change to auxin distribution within anther tissues. This could lead to transcriptional responses, thus activating the transcription of the NAC transcription factors NST1 and NST2, which in turn activating the expression of the downstream transcription factors, such as MYB103 and MYB85. This allows regulation the expression of the secondary wall biosynthetic genes, for example cellulose synthase complexes genes, CESA4, CESA7, CESA8, and xylan-reducing end sequence synthesis gene GAU12, thereby controlling the proper formation of secondary thickening in anther endothecium, and finally leading to breakage of the stomium and release of pollen grains from the locules during dehiscence. Arrows with dotted lines are used for speculative relationship, while 
arrows with solid lines represent experimental results.

\section{Supplementary Data}

Supplementary Fig. S1 The morphology of wild-type and three independent amiR-RUS4 lines.

Supplementary Fig. S2 Analysis of Arabidopsis RUS4 T-DNA insertion lines.

Supplementary Fig. S3 GUS staining of various tissues of RUS4pro:GUS transgenic plants.

Supplementary Fig. S4 The relationship between RUS4 and MYB26.

Supplementary Fig. S5 Stamen stained for secondary thickening with acridine orange/ethidium bromide and visualized by confocal microscopy.

Supplementary Fig. S6 Comparison of the secondary thickening in the stems and leaves of the RUS4 overexpression lines and wild-type.

Supplementary Fig. S7 The effect of overexpressing NST1 and NST2 in amiR-RUS4 genetic background.

Supplementary Table S1 Primers used for cloning and quantitative RT-PCR.

Supplementary Table S2 Pairwise protein sequence alignment

Two-protein BLAST alignments of RUS1, RUS2 to RUS4 using EMBOSS Needle

(http://www.ebi.ac.uk/Tools/psa/emboss_needle/). 


$$
\text { A }
$$

Hybridization energy: $-34.96 \mathrm{kcal} / \mathrm{mol}$ (85.98\%)

Target gene $5^{\prime} \rightarrow 3^{\prime} / 1374-1394$ aTCGGGCATCGTTATTTGTCT amiRNA (rev. complement)/1-21 aTGGGGCATCGTTATTTGTCA
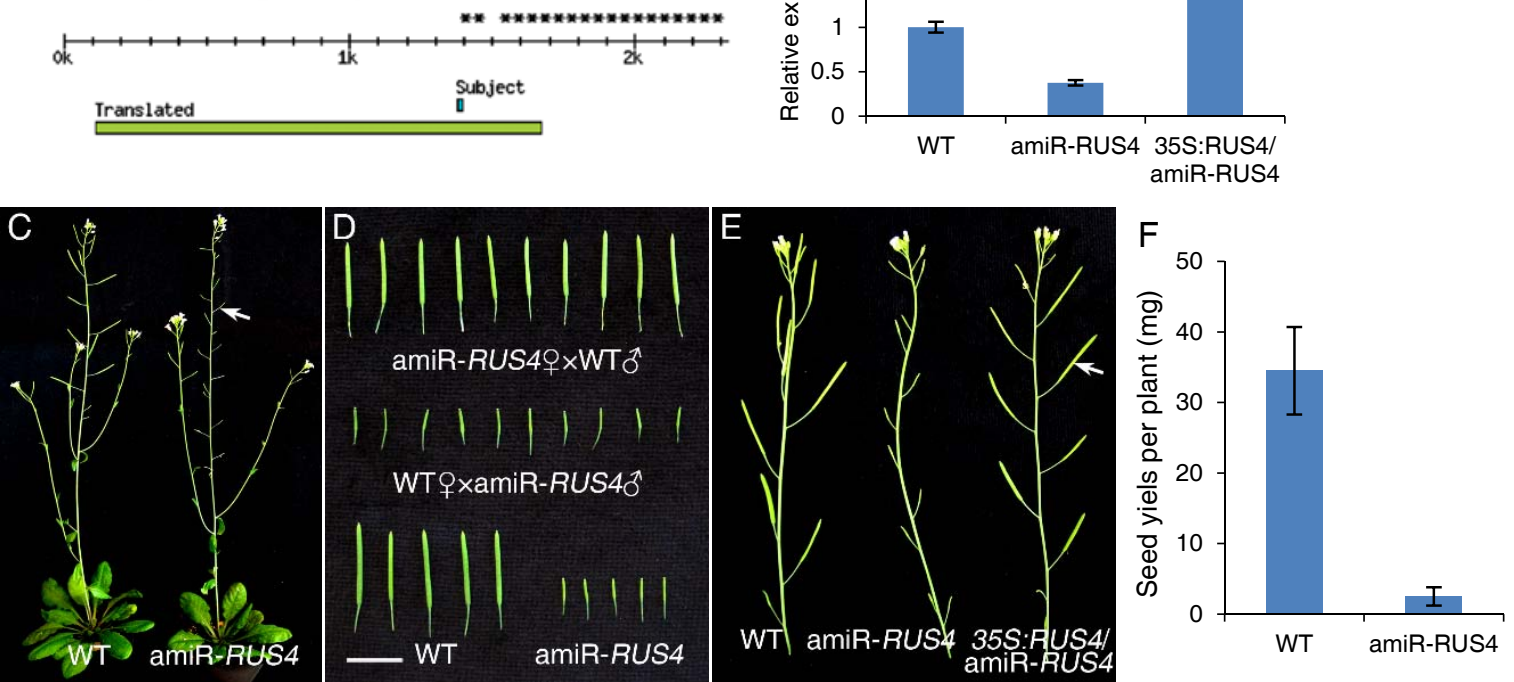

Fig. 1 

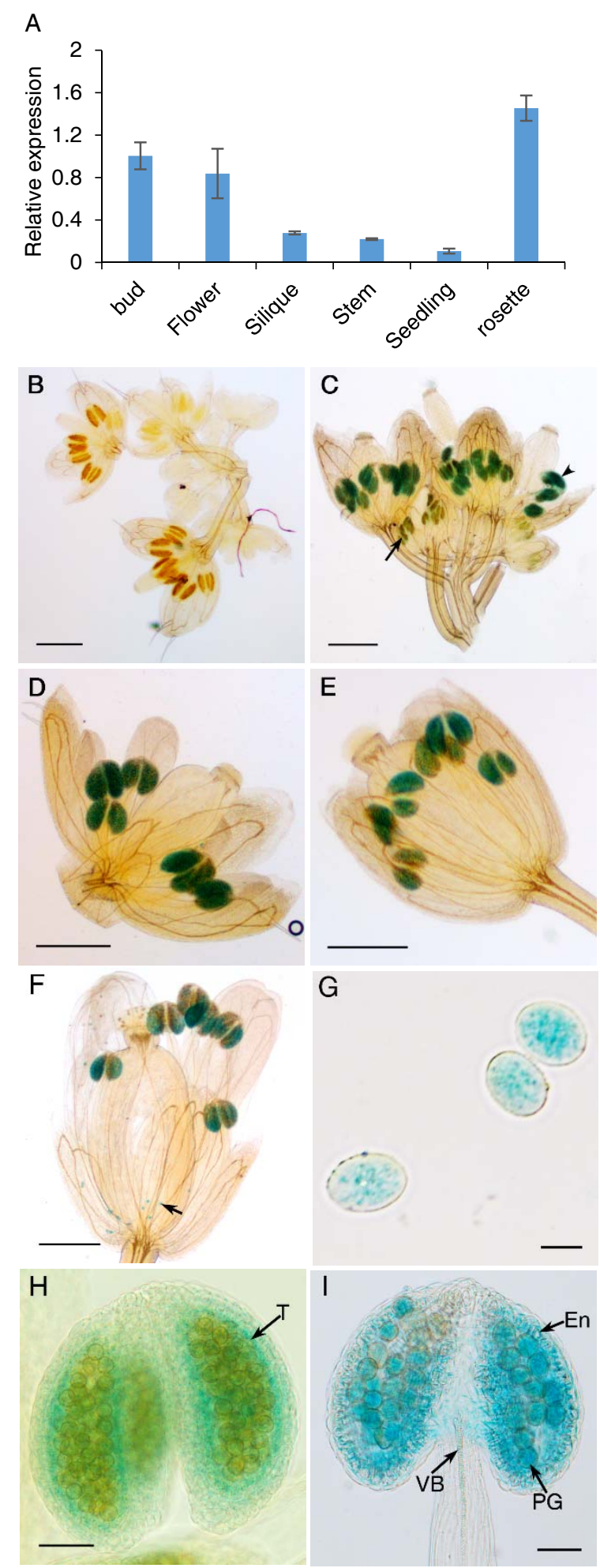

Fig. 2 


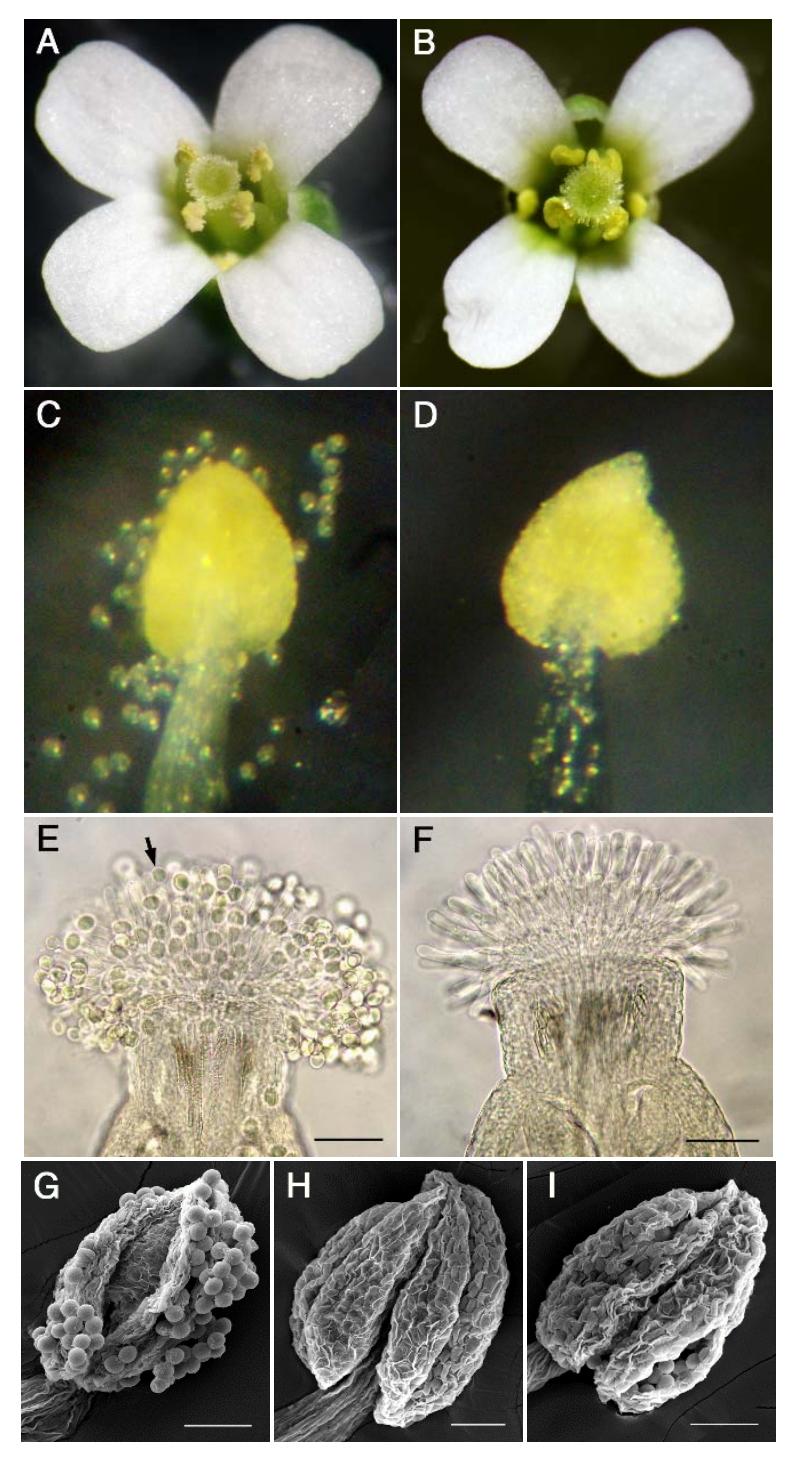

Fig. 3 


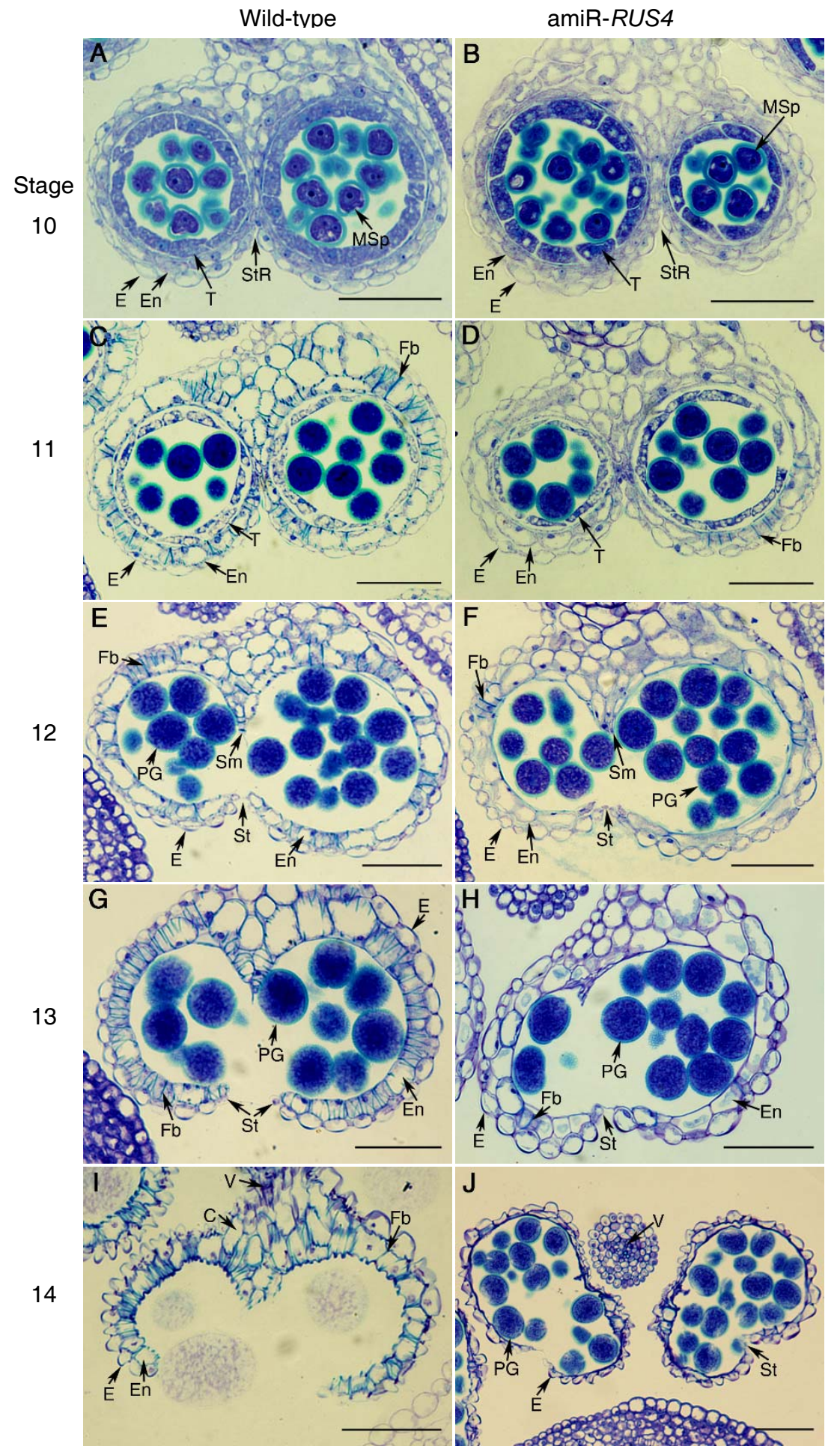

Fig. 4 


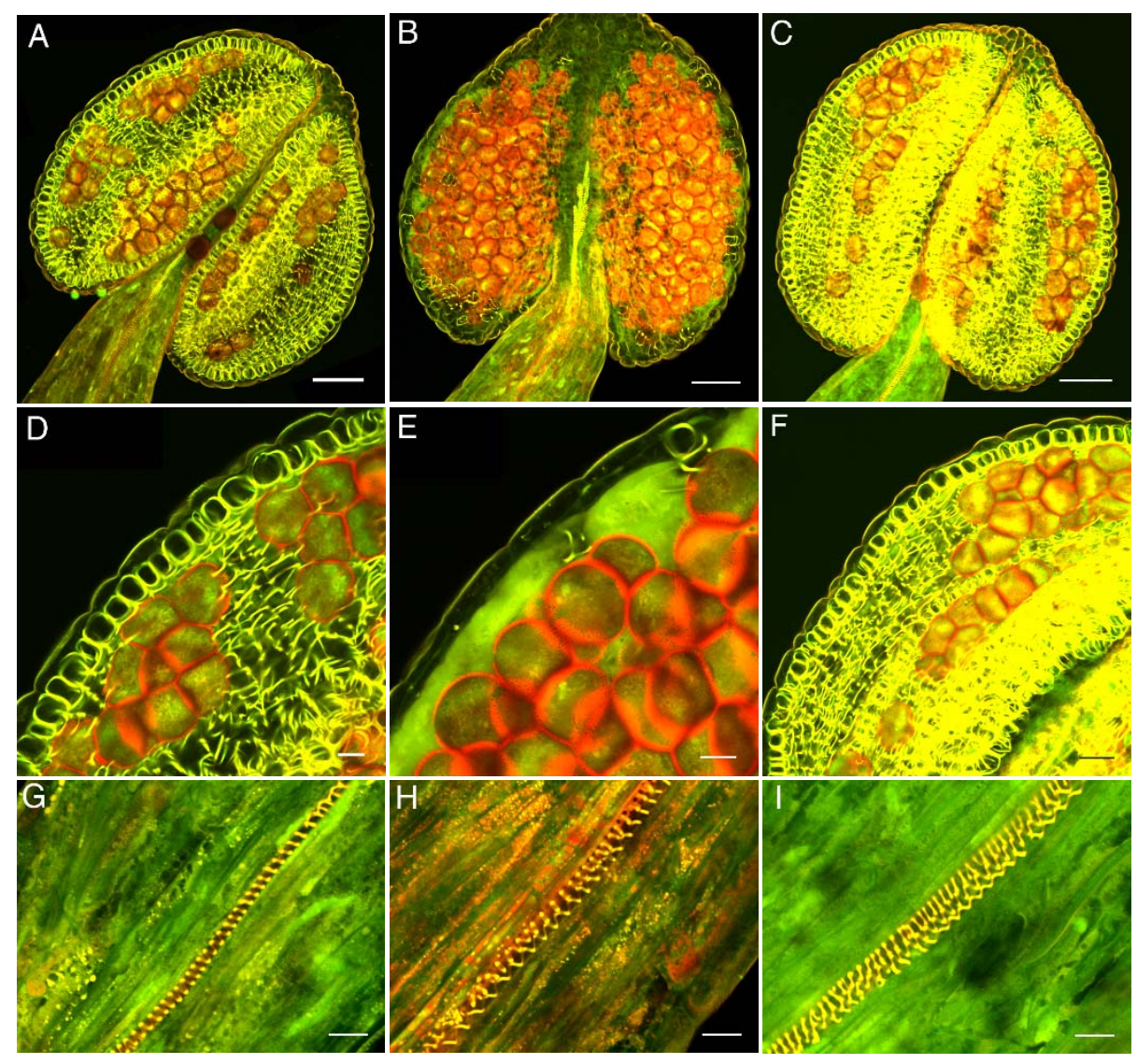

Fig. 5 


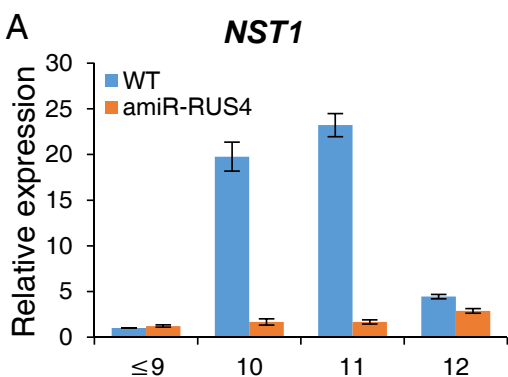

Flower developmental stage

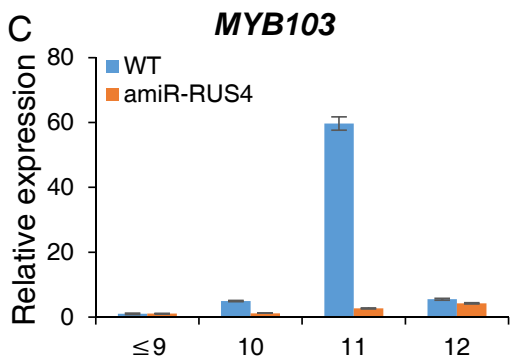

Flower developmental stage

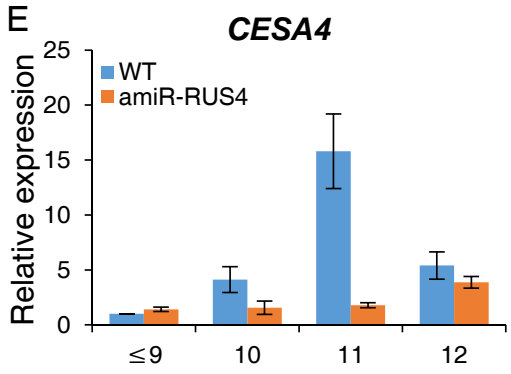

Flower developmental stage

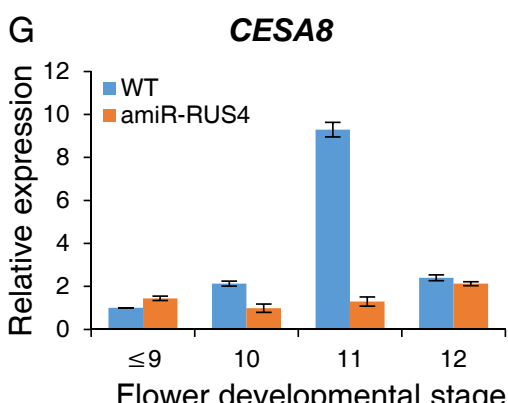

Flower developmental stage

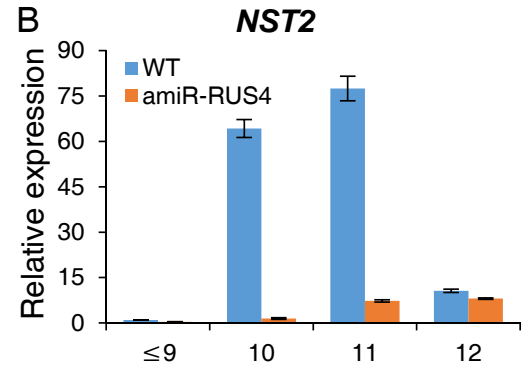

Flower developmental stage

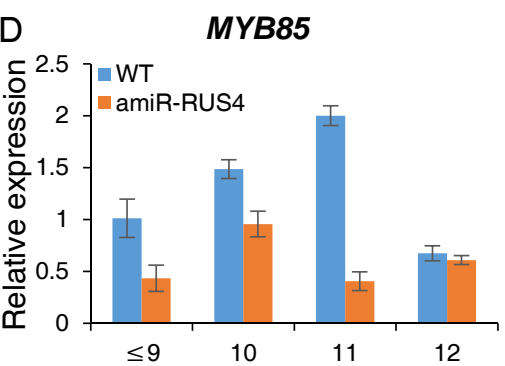

Flower developmental stage

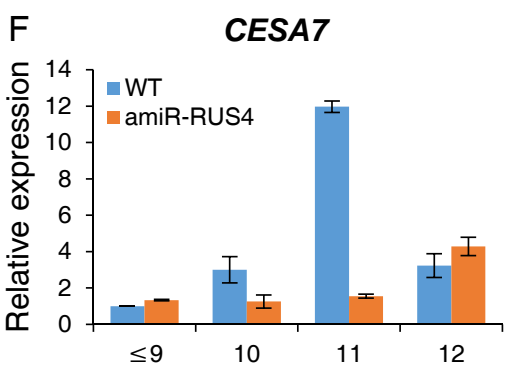

Flower developmental stage

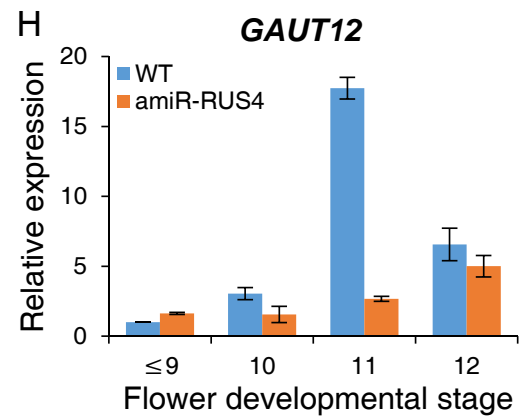

Fig. 6 

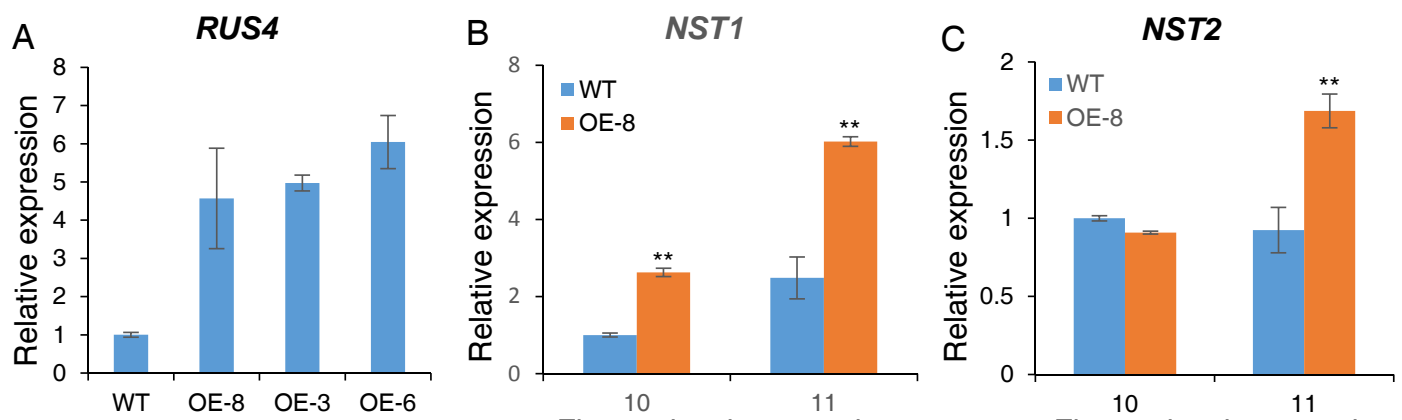

Flower developmental stage

Flower developmental stage

D

MYB103

E MYB85
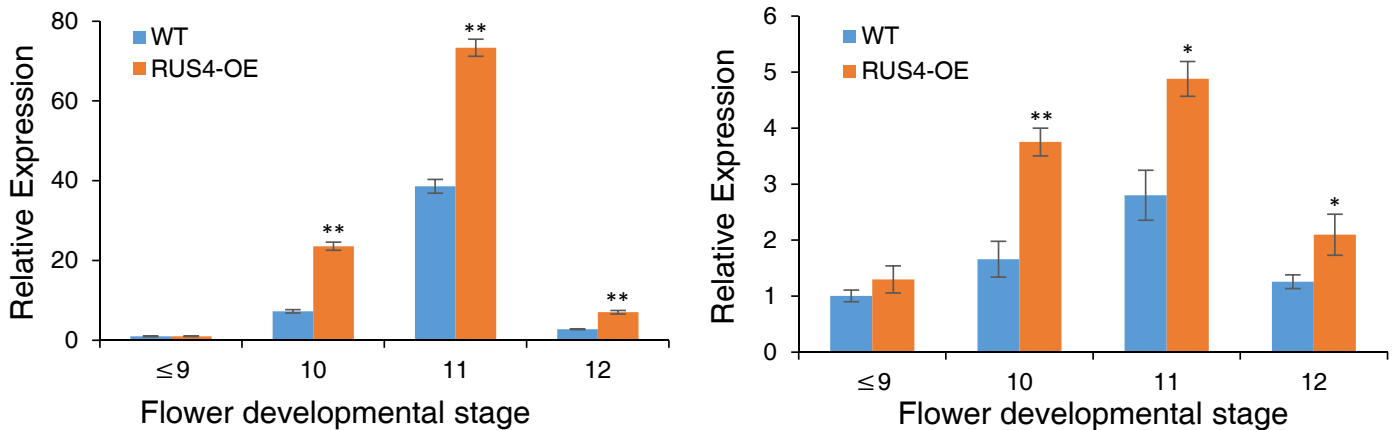

Fig. 7 


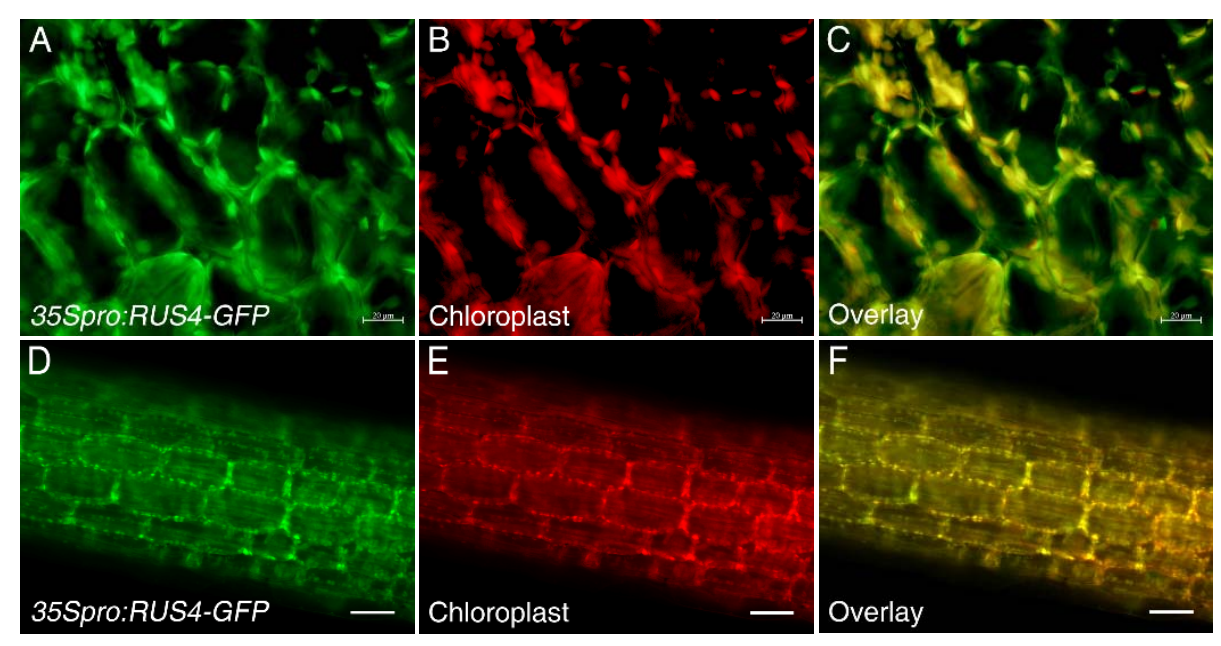

Fig. 8 


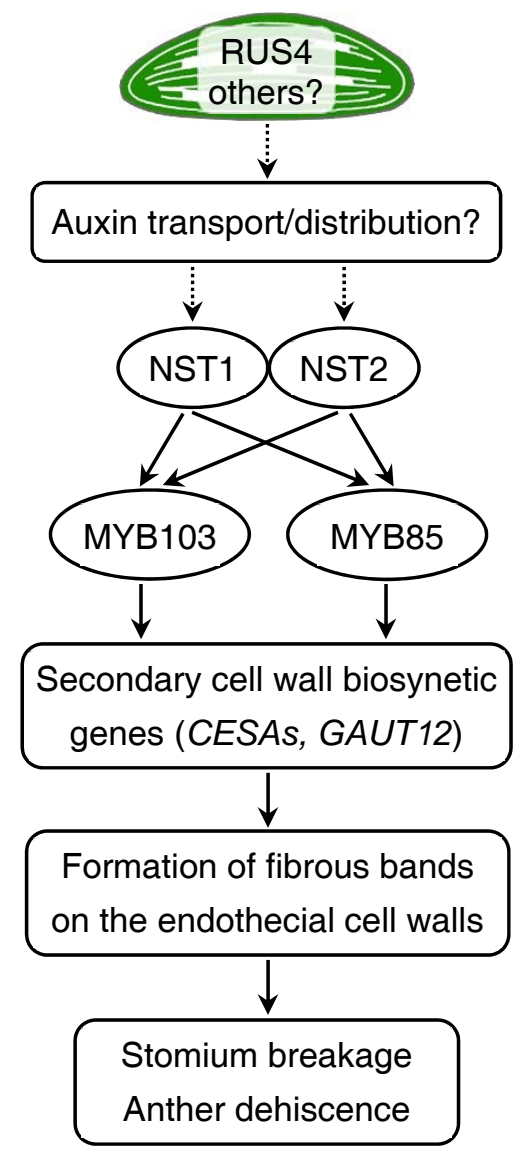

Fig. 9 